\section{Prevalência de sobrepeso e obesidade em crianças pré-escolares matriculadas em duas escolas particulares de Recife, Pernambuco}

\section{Overweight and obesity prevalence among preschool children of two private schools in Recife in the State of Pernambuco}

Gisélia Alves Pontes da Silva 1

Geni Balaban 2

Maria Maia V. Freitas 3

Joana Darc Santana Baracho 4

Eulália Maria M. Nascimento 5

1-5 Departamento Materno-Infantil. Centro de Ciências da Saúde. Universidade Federal de Pernambuco. Av. Moraes Rêgo, s. n, Bloco A. Recife, PE. Brasil. CEP 50.670-420. Tel / Fax (81) 3271. 8514

\begin{abstract}
Objectives: to determine overweight and obesity prevalence of preschool children in two private schools in Recife.

Methods: cross-sectional study of 230 preschool children enrolled in two private schools in Recife, in 2000. Overweight was defined as weight-for-height between +1 and $+2 z$ scores, and obesity as weight for-height above +2 zcores.

Results: prevalence rates were $22,6 \%$ for overweight and 11,3\% for obesity. Among female children, overweight prevalence was $27,0 \%$ and obesity prevalence was 9,8\%. Among males, overweight prevalence was $17,6 \%$ and obesity prevalence was 13,0\%.

Conclusions: overweight and obesity prevalence were high among preschool children from high income families. Pediatricians must be aware of the increasing prevalence of childhood obesity to be able to prevent, diagnose and treat this condition.
\end{abstract}

Key words Obesity, Child, preschool, Prevalence

\section{Resumo}

Objetivos: determinar a prevalência de sobrepeso e obesidade entre pré-escolares de duas escolas particulares em Recife.

Métodos: realizou-se um estudo de corte transversal com 230 pré-escolares matriculados em duas escolas particulares de Recife, no ano de 2000. Foram classificadas como portadoras de sobrepeso as crianças com índice pesolestatura entre $+1 a+2$ escores $z e$, como obesas, aquelas com pesolestatura acima de +2 escores $z$.

Resultados: as prevalências de sobrepeso e obesidade foram de 22,6\% e 11,3\%, respectivamente. Entre as crianças do sexo feminino, a prevalência de sobrepeso foi de 27,0\% e a de obesidade foi de 9,8\%. No sexo masculino, a prevalência de sobrepeso foi de $17,6 \%$ e a de obesidade foi de 13,0\%.

Conclusões: as prevalências de sobrepeso $e$ obesidade foram elevadas nesse grupo de pré-escolares, provenientes em sua maioria de famílias de classe socioeconômica média e alta. O pediatra deve estar atento para a crescente prevalência da obesidade infantil, a fim de atuar na prevenção, diagnóstico e tratamento dessa condição.

Palavras-chave Obesidade, Pré-escolar, Prevalência 


\section{Introdução}

A obesidade é um distúrbio nutricional traduzido por um aumento de tecido adiposo, resultante do balanço positivo de energia na relação ingesta-gasto calórico, que freqüentemente leva a prejuízos de saúde. ${ }^{1}$

$\mathrm{O}$ excesso de peso na criança predispõe às mais variadas complicações, abrangendo as esferas psicossocial, pois há isolamento e afastamento das atividades sociais devido à discriminação e à aceitação diminuída pela sociedade, e orgânica, uma vez que pode acarretar problemas respiratórios, diabetes mellitus, hipertensão arterial, dislipidemias, entre outros distúrbios. ${ }^{1,2}$

A obesidade infantil é um tema de interesse crescente devido ao aumento observado na sua prevalência. Odgen et al. 3 verificaram que a prevalência do sobrepeso em pré-escolares norte-americanos do sexo feminino aumentou entre 1971 e 1974 e 1988 e 1994, passando de 5,8\% para 10,8\%. Em nível nacional, Monteiro et al. 4 relataram uma prevalência da obesidade em menores de cinco anos variando de $2,5 \%$ entre os mais pobres, e $10,6 \%$ no grupo economicamente mais favorecido. Na região Nordeste, houve um aumento na prevalência da obesidade em crianças menores de cinco anos, provenientes de famílias de baixa renda, de 2,5\% em 1989 para 4,5\% em 1996.5 Um estudo realizado em Recife encontrou uma prevalência de sobrepeso no quarto e quinto anos de vida de $12,8 \%$ e $14,7 \%$, nos sexos masculino e feminino, respectivamente, entre crianças atendidas num consultório pediátrico privado. 6

A avaliação antropométrica é comumente utilizada para o diagnóstico de obesidade em estudos epidemiológicos, por consistir em procedimento simples, não invasivo, rápido e barato. $7,8 \mathrm{O}$ peso relativo à estatura, em tese, reflete as variações tanto no tecido adiposo quanto na massa magra dos indivíduos. Entretanto, em crianças de pequena idade, índices peso/estatura elevados geralmente não se devem ao aumento da massa muscular, mas sim à obesidade. ${ }^{9}$ As medidas das pregas subcutâneas, apesar de melhor refletirem a quantidade de tecido adiposo, estão mais sujeitas aos erros de medição. 10

A detecção de alterações na composição corporal durante a infância é importante, por permitir uma intervenção precoce e prevenir as complicações da obesidade. As modificações nos padrões alimentar e de atividade física, em geral, são mais aceitas pelas crianças e os hábitos alimentares são fixados neste período. ${ }^{11} \mathrm{O}$ presente estudo teve como objetivo determinar as prevalências de sobrepeso e obesidade em crianças pré-escolares matriculadas em duas escolas privadas da cidade do Recife.

\section{Métodos}

Realizou-se um estudo de corte transversal na Escola Fazer Crescer, localizada no bairro do Rosarinho, e na Escola Encontro, localizada no bairro das Graças, ambas escolas da rede privada de ensino, atendendo crianças das classes média e alta de Recife, PE, Brasil. A população de estudo constou de 250 crianças, correspondente ao total de alunos na faixa etária de dois a cinco anos de idade, de ambos os sexos, matriculados nessas escolas durante o ano letivo de 2000.

Em relação ao tamanho da amostra, considerando uma prevalência de $10 \%$ para obesidade, ${ }^{4}$ um power de $80 \%$ e um nível de significância de 5\%, estimouse um n de 164. Contudo, foi decidido estudar o total da população, ao invés de uma amostra, para dar mais consistência à análise.

Antes da coleta dos dados houve contato com a direção das escolas e sensibilização de professores e pais, com esclarecimento sobre os procedimentos a serem realizados e os objetivos do estudo. Os pais ou responsáveis autorizaram a participação dos seus filhos através de um formulário de consentimento informado. O estudo foi aprovado pela Comissão de Ética em Pesquisa do Centro de Ciências da Saúde da Universidade Federal de Pernambuco.

As crianças foram pesadas sem sapatos ou meias, vestindo os uniformes da escola, composto de camisa de malha e bermuda, numa balança digital eletrônica com capacidade para até $150 \mathrm{~kg}$, da marca Plenna, com precisão de $0,1 \mathrm{~kg}$, conforme recomendação da World Health Organization (WHO).12 A mesma foi colocada sobre uma superfície rígida e os alunos foram pesados em pé, com os membros ao longo do corpo, posicionados no centro da balança, olhando para frente. 7

A estatura foi medida com o auxílio de uma fita métrica de 1,5m, não distensível, com marcação de $0,1 \mathrm{~cm}$, fixada à parede, e de um esquadro de acrílico colocado sobre o topo da cabeça da criança, a fim de se obter um ângulo reto com a parede durante a leitura. Os alunos foram orientados a permanecerem eretos, com a cabeça posicionada de modo que o Plano de Frankfurt (o qual passa pela órbita e pelo meato auditivo externo) ficasse horizontal, joelhos esticados, pés juntos, braços soltos ao longo do corpo e com os tornozelos, glúteos e ombros em contato com a parede. ${ }^{7}$ Cada medida antropométrica foi realizada em duplicata, utilizando-se a média dos valores na análise dos dados, com o objetivo de serem minimizados os erros de medição.

Sobrepeso foi definido como índice peso/estatura entre +1 e +2 escores $z$, e obesidade como 
peso/estatura maior que +2 escores z. As curvas de peso para estatura do National Center for Health Statistics (NCHS) e WHO foram usadas como referência. 12

As perdas ocorridas (20 crianças) deveram-se à falta de autorização dos pais ou responsáveis ou à recusa de algumas crianças, principalmente as mais jovens, a submeterem-se às medidas antropométricas.

O software Epi-info, versão 6.0 do Center for Disease Control, foi utilizado para o armazenamento e análise dos dados. Inicialmente houve a abertura do banco de dados, com a entrada das seguintes informações: registro, data de nascimento, data da coleta de dados, peso e altura. As medidas antropométricas foram processadas através do software Epinut, que utiliza os padrões de referência do NCHS. As prevalências de sobrepeso e obesidade foram calculadas por proporção, e as diferenças entre os sexos analisadas através do teste do quiquadrado, com $p<0,05$. Foram também calculados os intervalos de confiança de $95 \%$ em torno de cada prevalência.

\section{Resultados}

Estudou-se um total de 230 crianças, sendo 108 (47\%) meninos e 122 (53\%) meninas. Observou-se sobrepeso em 52/230 crianças, uma prevalência de 22,6\% (IC95\%: 17,4 - 28,6); em 26/230 das crianças identificou-se obesidade, uma prevalência de 11,3\% (IC95\%: 7,5 - 16,1) (Figura 1).

\section{Figura 1}

Prevalência de sobrepeso e obesidade em pré-escolares de duas escolas particulares. Recife, PE, 2000.

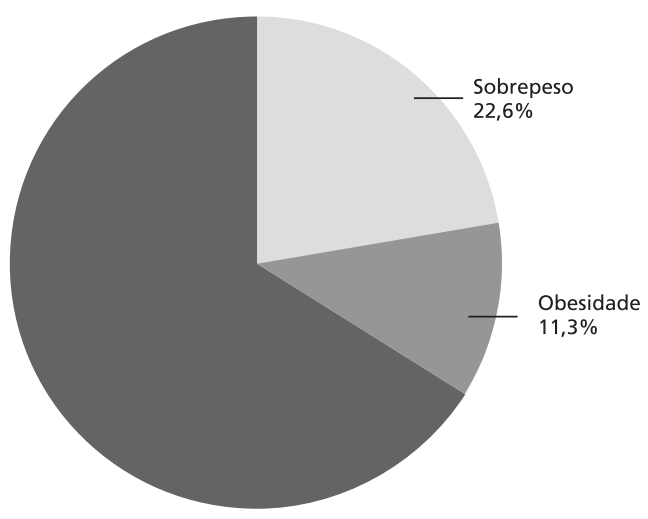

Entre as do sexo masculino 19/108 (17,6\%; IC95\%: 10,9 - 26,1) apresentaram sobrepeso, e 14/108 (13,0\%; IC95\%: 7,3 - 20,8) obesidade. Entre as do sexo feminino, 33/122 (27,0\%; IC95\%: 19,4 $35,8)$ apresentaram sobrepeso, e 12/122 (9,8\%; IC95\%: 5,2 - 16,5) obesidade. Não houve diferença estatisticamente significante entre as prevalências de sobrepeso nos sexos masculino e feminino $\left(\chi^{2}=\right.$ $2,41 ; p=0,12)$. A diferença entre as prevalências de obesidade nos sexos masculino e feminino também não teve significância estatística $\left(\chi^{2}=0,29 ; \mathrm{p}=\right.$ $0,59)$.

\section{Discussão}

Para o diagnóstico da obesidade vários métodos têm sido utilizados, sendo a avaliação antropométrica um método de fácil realização, de baixo custo, de boa correlação com a composição corporal e de alta reprodutibilidade para estudos epidemiológicos, quando comparada com outros métodos de determinação da gordura corporal, como a densitometria e a tomografia, os quais são caros e de difícil execução, pois exigem aparelhos sofisticados e qualificação profissional. 13

Uma elevada prevalência de sobrepeso $(22,6 \%)$ foi verificada entre os pré-escolares estudados, semelhante a dos países desenvolvidos. Bundred et al. 14 relataram uma prevalência de sobrepeso de 26,3\% em pré-escolares britânicos. Entre as crianças do sexo masculino, a prevalência de sobrepeso foi de $17,6 \%$, enquanto que no sexo feminino foi de $27,0 \%$. Um estudo realizado com crianças atendidas num consultório privado de Recife demonstrou prevalências de sobrepeso de $12,8 \%$ e $14,7 \%$, nos sexos masculino e feminino, respectivamente, entre o quarto e quinto anos de vida; mas os autores não utilizaram a mesma definição de sobrepeso/obesidade do presente estudo, o que deve ser levado em conta na interpretação dos resultados obtidos. 6

A prevalência de obesidade em nosso estudo $(11,3 \%)$ foi semelhante à relatada por Monteiro et al. 4 para crianças pertencentes às classes socioeconômicas mais favorecidas, em nível nacional $(10,6 \%)$. Bundred et al. ${ }^{14}$ encontraram uma prevalência de obesidade de 9,2\% entre crianças britânicas menores de cinco anos.

No presente estudo, embora não tenham sido analisadas variáveis socioeconômicas, o fato das crianças, em idade pré-escolar, freqüentarem escolas particulares sugere que são oriundas de famílias de maior poder aquisitivo.

Diferentes resultados têm sido encontrados ao se 
estudar a relação entre prevalência de obesidade e nível socioeconômico. Strauss e Knight, 15 estudando crianças de até oito anos de idade, em Nova Jersey, verificaram que aquelas com mães obesas, de baixo nível socioeconômico e baixo estímulo ao aprendizado, tinham elevado risco para desenvolvimento da obesidade. Sakamoto et al. 16 demonstraram haver marcante correlação direta entre a renda familiar e a prevalência de obesidade entre crianças tailandesas. Tem sido descrito que na América Latina a obesidade infantil tende a ser mais prevalente nas áreas urbanas e em famílias com nível socioeconômico e de escolaridade materna mais elevada. 17

Monteiro et al.,4 a partir de inquéritos nacionais de 1974, 1975 e 1989, afirmaram que a prevalência da obesidade aumenta de acordo com a renda. Em 1989, as prevalências de obesidade nas regiões Sul $(7,2 \%)$ e Sudeste $(6,2 \%)$ eram mais do que o dobro da observada na região Nordeste (2,5\%), ficando as regiões Norte e Centro-Oeste em situação intermediária. 5

Post et al.,18 estudando crianças com um ano de idade de duas coortes de base populacional no sul do
Brasil, também verificaram que a obesidade apresentou uma tendência crescente com o nível de renda familiar. Entre crianças pertencentes às famílias de renda maior, a proporção de obesos era em torno de $8 \%$ comparada com $2,5 \%$ nas pertencentes às famílias de menor renda.

A detecção do excesso de gordura durante a infância é importante, por permitir uma intervenção precoce e evitar a instalação de suas complicações. Quanto mais idade tiver a criança e maior for o excesso de peso, mais difícil será a reversão do quadro, pelos hábitos alimentares incorporados e pelas alterações metabólicas instaladas. 5

Ao contrário do tratamento da obesidade no adulto, que envolve necessariamente uma perda de peso, para uma criança ainda em crescimento pode ser necessária apenas a manutenção de seu peso enquanto continua a crescer, permitido que a mesma progressivamente se adeqüe à estatura.13 Dessa maneira, percebe-se a importância da atuação do pediatra na detecção e tratamento dessa condição ainda na infância, devendo a avaliação nutricional ser prioritária na consulta pediátrica.

\section{Referências}

1. Taddei JAAC. Epidemiologia da obesidade na infância. Pediatr Mod 1993; 29: 111-5.

2. Damiani D, Carvalho DP, Oliveira RG. Obesidade na infância: um grande desafio. Pediatr Mod 2000; 36: 489-528.

3. Ogden CL, Troiano RP, Briefel RR, Kuczmarski RJ, Flegal KM, Johnson CL. Prevalence of overweigth among preschool children in the United States, 1971 through 1994. Pediatrics 1997; 99: 1-7.

4. Monteiro CA, Mondini L, Souza ALM, Popkin BN. Da desnutrição para a obesidade: a transição nutricional no Brasil. In: Monteiro CA. Velhos e novos males da saúde no Brasil - A evolução do país e suas doenças. São Paulo: Hucitec; 1995. p. 247-55.

5. Escrivão MAMS, Oliveira FL, Taddei JAAC, Lopez A. Obesidade exógena na infância e na adolescência. J Pediatr [Rio de Janeiro] 2000; 76 Supl 3: 305-10.

6. Ferrreira OS, Batista Filho PN, Charifker H, Coelho EF, Lima GMS, Alves JGB. Sobrepeso em crianças atendidas em um consultório pediátrico privado do Recife. Pediatria [São Paulo] 1984; 6: 69-73.

7. Gibson RS. Anthropometric assessment of growth. In: Gibson RS. Principles of nutritional assessment. Oxford: University Press; 1990. p. 155-62.

8. Rolland-Cachera MF, Sempé M, Guilloud-Bataille M, Patois E, Péquignot-Guggenbuhl, Fautrad V. Adiposity indices in children. Am J Clin Nutr 1982; 36: 178-84.
9. Mondini L, Monteiro CA. Relevância epidemiológica da desnutrição e da obesidade em distintas classes sociais: métodos de estudo e aplicação à população brasileira. Rev Bras Epidemiol 1998; 1: 1-10.

10. Freedman DS, Srinivasan SR, Valdez RA, Williamson DF, Berenson GS. Aumento do peso relativo e do tecido adiposo em crianças, durante duas décadas: o estudo de Bogalusa. Pediatrics 1997; 1: 504-14.

11. Dietz WH. Periods of risk in childhood for development of adult obesity - What do we need to learn? J Nutr 1997; 127: 1884-6.

12. WHO (World Health Organization). Physical status: the use and interpretation of antropometry: report of a WHO Expert Committee. Geneva: The Organization; 1995.

13. Zlochevsky ERM. Obesidade na infância e adolescência. Rev Paul Pediatr 1996; 14: 124-33.

14. Bundred P, Kitchiner D, Buchan I. Prevalence of overweight and obese children between 1989 and 1998: population based series of cross sectional studies. BMJ 2001; 322: 326-8.

15. Strauss RS, Knight J. Influence of the home environment on the development of obesity in children. Pediatrics; 1999; 103: 85-9.

16. Sakamoto N, Wansorn, Tontisirin K, Marui E. A social epidemiologic study of obesity among preschool children in Thailand. Int J Obes Relat Metab Disord 2001; 25: 389-94. 
17. Martorell R, Khan LK, Hughes ML, Grummer-Strawn LM. Obesity in Latin American women and children. J Nutr 1998; 128: 1464-73.

18. Post CL, Victora CG, Barros FC, Horta BL, Guimarães PRV. Desnutrição e obesidade infantis em duas coorte de base populacional no Sul do Brasil: tendências e diferenciais. Cad Saúde Pública 1997; 12: 49-57.

Recebido em 6 de dezembro de 2002

Versão final reapresentada em 23 de junho de 2003

Aprovado em 3 de julho de 2003 